\title{
Catalysis on nano-carbon materials: Going where to?
}

\author{
Dang Sheng Su, ${ }^{1,2}$ Siglinda Perathoner ${ }^{3}$ and Gabriele Centi ${ }^{3}$
}

1 Shenyang National Laboratory for Materials Science, Institute of Metal Research, Chinese Academy of Science, 72 Wenhua Road, Shenyang 110006 (China)

2 Department of Inorganic Chemistry, Fritz Haber Institute of the Max Planck Society, Faradayweg 4-6, 14195 Berlin (Germany), dangsheng@fhi-berlin.mpg.de

3. Dipartimento di Chimica Industriale ed Ingegneria dei Materiali, University of Messina and INSTM/CASPE (Laboratory of Catalysis for Sustainable Production and Energy), V.le F. Stagno D'Alcontres 31, 98166 Messina (Italy), centi@unime.it

\begin{abstract}
This introductory contribution to the special issue of Catalysis Today is dedicated to selected contributions presented at the Carbocat-IV (Carbon for Catalysis) Symposium held in Dalian (China) on November 7-10 (2010). The introduction first shortly overviews the main reasons for the interest on carbon materials for catalysis and the elements of novelty discussed on the contributions of this issue, and then provide a concise outline of the general trends and developments in this field to give a glimpse on the progresses in the field, and on the perspectives of this exciting area of catalysis.
\end{abstract}

Keywords: nano-carbon, catalysis on carbon, nanostructured carbon-based materials, carbon hierarchy

\section{Introduction}

Active carbon (AC) is known for long time as an excellent support for catalytic nanoparticles [1-3] and is still largely used industrially, particularly in selective hydrogenation reactions. Among the many attracting properties it may be cited the high surface area, the possibility of a fine tuning of the porosity, the robustness and chemical inertness (except in the presence of oxygen at high temperature), the possibility to tune the graphitic character (and thus the conductivity properties), the presence of heteroatoms and inorganic elements (deriving from the starting material to prepare the active carbon) which determine the surface character of the support (including hydrophilicity and interaction with the supported active phase), and not least the low cost. However, for long time due to the difficulty in the characterization of carbon materials, it was a lack of fundamental understanding of the surface chemistry of carbon used as support in catalysis and thus with a development mainly driven from a trial-and-error approach.

During the last decade, large progresses have been made in this direction, resulting in the opening of new possibilities in the use of AC as catalyst support for a variety of processes, and some of them are also applied in an industrial scale, such as: i) environmental protection area (volatile organic compounds 
oxidation, chlorinated compounds dehalogenation and advanced oxidation technologies such as catalytic wet-air oxidation); ii) selective oxidation processes (oxidation of glycerol etc. or heterogeneous Wacker-type catalysts for the oxidative carbonylation of methanol); iii) hydroprocessing and selective hydrogenation; iv) various applications for fine and specialty chemicals synthesized from base catalyzed reactions (for example, $\mathrm{Na}-, \mathrm{K}$ - and Cs-AC for N-alkylation of imidazolic rings), acid-catalyzed reactions, or reactions leading to CC bond formation (Suzuki, Heck, Sonogashira, and Ullmann reactions, etc.) [4].

Using AC as a catalyst support always provides unparalleled flexibility in tailoring their physical (surface area and porosity) and chemical (surface functional groups) properties. The surface chemistry of AC in term of surface functionalities (O-and N-containing groups, H-C species, S, P, and halogen functionalities, and other heteroatoms such as B) is known today in a much more detail, as well as how to tune these characteristics by secondary treatments (oxidation, halogenization, impregnation, dry mixing, and heat treatments) [5].

A second factor stimulating the research interest on carbon for catalysis is that carbon materials may be considered one of the first examples for the synthesis of tailored 1D and 2D nanostructures. As remarked in the editorial to a special issue of "ChemSusChem" on 20 Years of Carbon Nanotubes (CNTs) [6], their formation is known since 1976, while single-walled carbon nanotubes have been observed since 1993. However, the observation of "helical microtubules of graphitic carbon" under electron microscope by Iijima [7] is considered to be the practical start for the worldwide large scientific and industrial interest on CNTs which transformed later to the interest of developing tailored nanostructures for nanocarbon-based catalysts. The progresses on understanding of the growth mechanism of CNTs [8] improved their yields moving progressively towards their mass production, and also allowed to control the quality and nanostructure (number of walls, graphene sheet orientation, chirality, alignment, defect nature, etc.) of CNTs. Initially, the utilization of CNTs in catalysis led to a number of contrasting results due to their inhomogeneous characteristics [9]. The advances made in the understanding of characteristics, surface chemistry and presence of defect sites of CNTs, together with their availability in large amounts at reasonably low costs [10], allowed a significant progress in their application in preparing advanced catalysts. The rapid increase of the research on the synthesis of nanostructured carbon materials significantly extended the number of different carbon nanostructures available for catalytic uses (carbon nano-fibres, -tubes, -coils, -horns, diamond, -onion, graphene, etc.)[11-13]. The progress in controllable doping of these materials (particularly with $\mathrm{N}$ and $\mathrm{B}$ ) [14-17] and in tailored assembly of these low-dimensional carbon materials into threedimensional architectures (films, hollow spherical capsules, or hollow nanotubes) [18] has further stimulated the availability of materials and provided new ideas for their uses in the field of catalysis. For example, the direct growth of ordered arrays of carbon nano-tubes or -fibres (carpet-type) from nanopatterned catalyst arrays or using inorganic templates [19-21] opens new possibilities to develop advanced catalyst architectures or novel and efficient catalysts for microstructured reactors [22].

A third pushing factor for the interest on carbon-based catalysts is the worldwide need to develop more efficient approaches for advanced electrodes for more sustainable utilization of energy (from fuel cells 
to batteries, photoanodes and solar cells). The conductive properties of (some) carbon materials together with the possibility of fine tuning of their nanostructure are critical elements to realize advanced electrodes to meet the demanding expectation for more sustainable and efficient conversion and storage of energy [23,24]. Several research groups working in catalysis have moved to electrochemical field bringing back new expertise on catalysis, because carbon materials used in electrochemical devices exhibit catalytic behavior in addition to electrochemical properties, and which is very close to the interest for catalysis.

For these and other reasons, the application of advanced carbon-based materials was fast growing over the last decade. As a matter of fact, it was an exponential increase in the field of carbon and catalysis, particularly in nano and electro-catalytic aspects (Table 1) during the last decade. This was the motivation to start the series of symposia "Carbocat", with this issue collecting selected contributions presented at 4th International Symposium on Carbon for Catalysis, held in Dalian (China) on November 7-10, 2010. During this conference five major topics were discussed in over 100 contributions (between oral and posters): i) synthesis of novel structured carbons, ii) characterization and modeling, iii) carbon-based materials in catalytic reactions, iv) carbon for clean and sustainable energy, and v) new trends in carbon technologies for adsorption and environmental protection. Symposium Co-Chairmen were Xinhe Bao (Dalian Institute of Chemical Physics, China) and Dangsheng Su (Fritz-Haber-Institut der Max-Planck-Gesellschaft, Germany).

Previous meeting were held in Lausanne, Switzerland (2004), St. Petersburg, Russia (2006) and Berlin, Germany (2008). The next one (Carbocat-V) will be organized in Brixen, Italy, on June 28 to 30, 2012, and the Symposium Chairpersons will be Siglinda Perathoner, Candida Milone (University of Messina, Italy), and Laura Prati (University of Milan, Italy). Also this conference will be devoted to the fascinating world of carbon based materials for catalysis and the new possibilities for the design of advanced nano-architectures. Besides the discussion on the synthesis and application of carbon-based materials in catalytic reactions, emphasis will be also given to the major fields triggering recent research in this field, such as graphenebased catalysts and carbon based materials for clean and sustainable energy etc. These are emerging areas, together with advancement in the understanding and modeling of carbon-based catalysts.

There are several new advancements discussed in this issue. The contribution of Bao et al. shows how the confinement of FeN particles inside CNT leads to interesting properties for the synthesis of light olefins from syngas, which is a field of large current interest for the possibility of chemical valorization of coal. The contribution of Xiao et al. reports the synthesis of magnetically active mesoporous carbons. Besides the applications discussed in the paper, the combination of magnetic and catalytic sites offers new possibilities for an easy recovery of the catalysts (in slurry reactors) or to induce synergetic effects through modulation of the magnetic field. Su et al. reported the application of highly ordered carbon nanofibres in the oxidative dehydrogenation of ethylbenzene, which is an example of the new promising area of research on metal-free catalysis in redox reaction associated with the functional groups presented on the surface of carbon $[25,26]$. Prati et al. discussed how to tune the hydrophilic properties of carbon nanotubes, and the relevance for tuning the properties of supported Pd nanoparticles active in the oxidation for alcohols. Yuan et al. reported the use of ordered mesoporous carbon materials for the challenging reaction of selective dehydrogenation of propane 
to propylene. Liang et al. evidenced the role of oxygen surface functional groups in CNTs to control the size and reactivity of supported Pt particles for phenylacetylene hydrogenation. There are many interesting examples discussed in the contributions in this issue of Catalysis Today, which are not only limited to catalysis on carbon materials, but also some of other related areas, such as the use of these materials for drug delivery (Carabineiro et al.) and the development of advanced membranes (Karpacheva et al.). The understanding of these materials is also well represented by the contributions of Chen et al. on transient isotope experiments, of $\mathrm{Zhu}$ et al. on density functional theory modeling of the effect of $\mathrm{Ag}$ on the Nicatalyzed carbon formation, and of Bandosz et al. on the mechanism of adsorption of toxic gases. Synthesis of advanced carbon materials is still one of the major topics reported in literature, and well represented in this issue with the contributions of Huang et al. on the synthesis of aligned N-doped carbon nanotubes in a fluidized bed reactor, of Chen et al. of platelet carbon nanofibres, of Dai et al. of mesoporous carbon materials with tailored surface acidity and Podyacheva et al. of Pt nanoparticles supported on N-doped carbon nanofibres. Hydrogenation/dehydrogenation reactions on CNT supported Pd catalysts and phenol oxidation on cobalt/carbon xerogels, by Kim et al. and Wang et al., respectively, complete the topics discussed in this issue. There is thus an interesting panorama of the current trends in the field of carbonbased catalysts and a good progress update on the new developments in the field with respect to the Catalysis Today issue (Volume 150, issues 1-2, 2010) reporting selected contributions from the previous Carbocat-III symposium held in Berlin on 2008.

However, this is a very dynamic and active research field, as emerges also from the data reported in Table 1, and it may be thus important to outline also some of the general trends and developments in this field to complete the update and state-of-the-art. Although it is not the aim to provide here a full review, the following sections will shortly give a glimpse on the progress in the field, from a personal perspective, and on the outlooks of this exciting area of catalysis. The aspects highlighted will put the following contributions in a wider context and will allow the reader, particularly the younger ones, to have a better feeling of the opportunities offered from this research sector.

\section{Synthesis of novel structured carbon}

The synthesis of nano-carbons (we use here the term nano-carbons, because it is becoming a frequently used term to indicate nanostructured carbon materials) with controllable properties and their possible production at industrial scale are the prerequisite for applications and investigations as catalysts. The large effort dedicated in literature on these aspects is thus important. In 2010, the number of papers associate with the synthesis of carbon materials was about 2.7 times higher than those on carbon materials for catalysis, which in part were also mainly dedicated to the synthesis and characterization. The synthesis and characterization of novel structured carbon materials is thus an important topic, but it is necessary to shift now part of the attention on the catalytic reactivity of the developed materials to well defined and well characterized samples to avoid the problem of irreproducibility in the results, which were due to the large differences in the characteristics of apparently the same materials (carbon nanotubes, for example). 
Multi-wall carbon nanotubes (MWCNT) are still the main type of nano-carbon material studied for catalytic applications, which can be produced today in a larger scale at accessible costs by chemical vapour deposition (CVD) [8]. The residual catalyst impurities in the obtained MWCNTs are scarce and the purity of MWCNTs can be above $99.5 \%$, which is sufficient for most applications, but impurities may sometimes alter the results in catalytic applications. The production of single-wall CNTs (SWCNT) at an industrial scale, compared with that of MWCNTs, is instead underdeveloped. Although SWCNT are potentially preferable in a number of applications, the lack of mass production (at a reasonable cost) is still a limit for their usage. Although there are some recent progresses, a breakthrough in SWCNT production with controllable chirality at large scale is still missing [8]. This is thus an area in which an intensified effort is necessary. Also the production of specific nano-architectures, starting from the preparation of vertically aligned CNTs (carpets), is still at the laboratory scale. One possibility to reduce the costs is to use natural materials (either inorganic or organic) containing traces of iron as catalyst [27-29], but the reproducibility of the starting materials on a large (industrial) scale to have constant-quality products is a major issue. Therefore, except for MWCNT, the production on a larger scale of novel structured carbon materials is still a problem to extend the use of nanocarbon materials as catalysts.

Graphene, a honeycomb-like carbon sheet strictly of one atom thickness, is a material potentially of large interest to produce advanced catalysts, and the interest on which has been sharply raised from the Nobel awards in Physics 2010 to Andre Geim and Konstantin Novoselov "for groundbreaking experiments regarding the two-dimensional material graphene". However, current methods for production of graphene are still too expensive for catalytic applications. Chemical synthesis of graphene is currently the potentially cheaper alternative method for large scale. This method is based on the preparation of graphite oxide and subsequent chemical reduction to yield a suspension of graphene flakes [30]. However, the graphene obtained by this route still has many chemical and structural defects.

Unzipping CNT to graphene nanoribbons using either strong oxidants or catalytic nanoparticles [31,32] is also a promising method. The obtained graphene may be suitable for catalytic applications or for electrodes, although it is typically graphene sheets with few-layers. A range of nano-engineering possibilities are opened by extending these methods. For example, with the assistance of encapsulated Co particles, it is possible to achieve a precisely controllable cutting, repairing, and interconnecting of different CNTs [33]. A graphene nanoribbon can have a different chirality depending on the angle at which it is cut, and as a consequence it exhibits tunable energy gaps and one-dimensional edge states with unusual magnetic structure [34]. The graphene nanoribbon, depending on the width, length, chirality, and substrate, may thus show very different properties for supported metal particles, but the analysis of the data is obviously difficult due to the absence of precise characterizations of the materials.

Sub-nanometer supported metal clusters and alloys are novel highly challenging area of catalysts, because metal particles have unusual catalytic reactivity below around 1nm [35]. However, stabilization of these so-small particles is clearly difficult and challenging. Magnetic nanocenters in graphene nanoribbons or other nanocarbons are one of the attracting novel possibilities to stabilize these so-small nanoparticles. 
Ordered mesoporous carbons (OMCs, $2 \mathrm{~nm}<$ pore size $<50 \mathrm{~nm}$ ) is another still attracting research area. They can be prepared by hard and soft templating synthetic methods [36]. The hard templating method has advantages of simplicity and fidelity in producing the OMC, but the procedure is complicated, timeconsuming, and unsuitable for the mass production, because several steps are required to fabricate the structure matrices and then remove the siliceous template matrices under harsh chemical treatment conditions. Soft templating method uses phenolic resin and block copolymer PEO-PPO-PEO to prepare highly OMCs via organic-organic assembly of block copolymers and phenolic resins [37,38]. With this method, the large scale synthesis of OMC materials is viable [38]. Unfortunately, OMCs obtained by this way typically do not offer any macropore character (pore size $>50 \mathrm{~nm}$ ). By combining hard- and softtemplating methods, hierarchically ordered porous carbons with designed porosity on multiple length scales can be obtained $[39,40]$.

Carbon hierarchy is the combination of at least two nanocarbon (graphene, carbon nanotubes or nanofibers, nanodiamonds, nano-porous carbons or polymers) building blocks, and it always induces superior properties in comparison to those of the individual elements [41]. It is of critical importance to overcome possible mass transport limitations in catalysts, combining high surface area with good accessibility, but also to combine different specific properties, such as those of carbon nanotubes and nanofibres [42]. In electrocatalysis and in energy storage devices [23,24,43], a single component could not meet the high demanding requirements of batteries, supercapacitors or electrodes for fuel cells.

However, a general problem not well understood is the presence of several interfaces which may give discontinuities in transport and in chemical properties, and possible deterioration of the overall properties and the chemical stability [44]. While the number of examples on the synthesis of nice carbon hierarchic structures is continuously growing, the progresses in terms of increased performances are slow, because not enough attention is given to this interface problem, as well as the nano-level characteristics of the materials produced (defects, interfaces, etc.). This is the area on which the future research should focus to have a real progress in using these materials.

Catalysis requires not only large amounts of CNTs and CNFs with well-defined surface-chemical and mechanical properties, but also nanocarbons formed into larger objects to optimize the reaction process and allow effective contact with reacting matrices. Loose CNTs/CNFs are unsuitable as their suprastructural properties cannot be controlled. From the view point of chemical engineering, the use of nanoscopic catalysts is hampered in large-scale fixed-bed reactors because of the large pressure drop across the catalyst bed and the problems associated with handling and transport of these materials. Although a number of progresses have been made in this area recently, it is still an area in which an intensified effort is necessary.

\section{Characterization and modeling}

Significant advances have been made in this field during the last decade and there is a broad portfolio of techniques for the characterization of nanocarbon materials today. However, due to the intrinsic difficulties in the characterization of these materials and the need to use well defined systems for catalysis, it 
may be useful to adopt a standard characterization protocol for nanocarbons [45]. A typical simplification is that carbon, different from other supports such as oxides, is inert, and does not participate as co-catalyst or induce significant modifications in the nature of supported active particles. There are increasing evidences showing that both of them are wrong assumptions. For example, a microcalorimetry study of the $\mathrm{CO}_{2}$ uptake on iron nanoparticles supported over on N-doped CNTs [46] (these are electrocatalysts able to selectively reduce $\mathrm{CO}_{2}$ to isopropanol, a very challenging reaction [47,48]) reveals that there are two types of chemisorption sites on the catalyst: irreversible sites $(280 \mathrm{~kJ} / \mathrm{mol})$ at the uncoordinated sites of the facets and reversible sites $(120 \mathrm{~kJ} / \mathrm{mol})$ at the hydrated oxide surface of small nanoparticles. The latter sites are correlated to the enhancement of the catalytic performance. Therefore, specific sites are present at the interface between the catalyst nanoparticles and the carbon support. The N-doping is important in creating these sites.

It was also shown by calorimetry that the curvature of CNTs enhances the adsorption of aromatic compounds in comparison with a graphite surface [49]. For instance, CNTs adsorb toluene more strongly than graphite. In addition, the presence of oxygen groups on CNTs modifies the surface electron density and thus the interaction mechanism with the adsorbates. It is necessary, however, to make a next step and use adsorption microcalorimetry to characterize the surface chemical properties of nanocarbons in conditions close to catalytic reaction. This is a challenging, but possible task [50]. For example, this method was used to quantify the different adsorption sites and correlate with the catalytic activity of CNT catalysts in the oxidative dehydrogenation of propane [50].

Significant advances have been also made in the understanding of how to create, characterize and control the surface sites present in nanocarbons. The stability and transformation of various O- and Ncontaining functional groups at high temperature is of relevance for their application in reactions at high temperature. The dynamic property of surface as a function of temperature is of significance to understand the catalytic behavior of nanocarbons. Nitrogen-functionalized carbons have been explored as catalyst or as catalyst support. The stability and transformation of various $\mathrm{N}$-containing functional groups at high temperature is thus of importance for reactions at high temperature, but it needs a combination of many advanced characterization techniques. For example, Arrigo et al. [51,52] investigated the dynamic nature of nitrogen functional groups on CNTs by means of temperature-programmed (TP) XPS (using synchrotron radiation) and TPD-MS. Synchrotron-based ambient pressure photoelectron spectroscopy (APPES) [53] is an unique tool to study the change of carbon surfaces in the presence of reactive environments and to establish correlations with the catalytic activity, for example the redox behavior of quinone groups on the carbon surface and their performances in the oxidative dehydrogenation of n-butane [54].

Electron tomography is another tool which is becoming critical for the characterization of nanocarbons and the localization of supported nanoparticles, for example, in the inner or outer surface of carbon nanotubes. The recently developed aberration-corrected (S)TEM has an improved resolution down to sub-Å. The elimination of Fresnel diffraction at the perimeter of the specimen allows a sharp imaging of surface structure of a catalyst [55]. In general, carbon materials could suffer electron beam radiation damage 
when a TEM is operated at high voltage at high electron dose. This damage is drastically minimized or even reduced to a minimum level at a voltage lower than $100 \mathrm{kV}$. The low-voltage electron microscopy, equipped with aberration-correctors to compensate the loss of resolution, provides a new possibility to study the point defects and doping of carbon materials [56]. Both of them are of essential important for understanding of carbon as catalyst and catalyst-support.

Parallel with the increased research interests on carbon as catalyst and catalyst-support is the increase of theoretical modeling and simulation of the reactivity of nanocarbons. A driving force for the theoretical computational studies may be the enormous interest on the chemical properties of graphene, especially those on the edges of the graphene sheet, as an emerging star in nanocarbon materials. However, molecular-level engineering of carbon-based catalysis cannot be successful until and unless the issues such as (re)active sites, doping effects, activation of reactant molecules on carbon and reaction micro-kinetics are clarified by first principle computational simulations [57].

In addition to understand by theoretical modeling the nature of interaction between carbon and supported metal particles [58] which creates bond length disorder deriving from interactions of the bottom metal layer in contact with the carbon surface [59], there is a number of intriguing aspects that are emerging. Qin and Liu [60] have shown by molecular dynamics simulations and density functional theory (DFT) calculations that $\mathrm{CuO}$ nanoparticles confined in carbon nanotubes have different reactivity with respect to those supported outside the carbon nanotubes. Zoberbier et al. [61] have also demonstrated that clusters of transition metals (W, Re, and Os), upon encapsulation within single-walled carbon nanotubes (SWNT) exhibit marked differences in their affinity and reactivity. Point defects in carbon nanotubes have a significant effect in anchoring sites of Au nanoparticles, as shown by DFT [62]. Other defect sites in carbon (specifically ordered mesoporous $\mathrm{C}$ ), such as sulfur, can equally play a critical role in the enhancement of the electrochemical stability and catalytic activity of supported Pt nanoparticles [63].

Shan et al. [64] showed that oxygen molecule dissociation is facilitated on carbon atoms neighboring a nitrogen dopant, with the dissociation barrier reducing from $2 \mathrm{eV}$ to $0.68 \mathrm{eV}$. The activation barrier can be further reduced to $0.03 \mathrm{eV}$ in the vicinity of a N-doped SW defect. Radovic [57] showed the different chemical nature and reactivity of carbon atoms at graphene edges and at nanotube ends, as well as at various types of defects within the basal plane of $\mathrm{sp}^{2}$-hybridized carbon materials.

These non-exhaustive examples show the richness of the chemistry of nanocarbon materials which open interesting perspectives in terms of application, and how theoretical modelling is a necessary tool for their understanding and possible applicable use.

\section{Catalytic uses}

Parallel with the growing knowledge on the synthesis and understanding of nanocarbon materials their application as advanced catalysts moved to more rational bases. The largest area of application still remains in clean and sustainable energy, and new opportunities are also offered in the chemical synthesis area. This is still an area growing slowly, and it is thus worthwhile to mention some of the possibilities offered from 
nanocarbon materials.

In fine chemicals transformations, and also in other applications when a complex mixture is present, it may be necessary to control the hydrophobic character of the support to limit side reactions and/or to control how the reactants chemisorb on the catalyst. Seo et al. [65] showed that it is possible to produce the carbon surface superhydrophobic property in a wide $\mathrm{pH}$ range (1-13) by covering carbon fibers with thin polydimethylsiloxane films. This is interesting in a number of applications, for example to limit water chemisorption on active sites etc. Lee et al. [66] covered MWCNT with polyaniline and showed that Pt-Ru nanoparticles supported on them have different characteristics with respect to those supported on bare MWCNT, where a Pt-rich core and a Ru-rich shell nanostructure is present. The catalytic activity for these materials is consequently different.

The presence of surface functional groups on nanocarbon materials also modifies the intrinsic reactivity. Soria-Sanchez et al. [67], investigated the reactivity of different nanocarbon materials in the catalytic oxidation of phenol in aqueous phase, and showed how the concentration of surface carboxylic surface groups influences the pathway of oxidation, leading to less formation of refractory short chain acids, like formic, malonic and oxalic acid during the reaction. Using graphene nanosheets, it is possible to stabilize sub-nano Pt clusters which show peculiar reactivity and high CO tolerance [68].

Functional groups presented on carbon surface are also showing interesting catalytic properties. Frank et al. [69] evidenced that $\mathrm{sp}^{2}$ carbon acts as a bifunctional catalyst in acrolein oxidation to acrilic acid. The nucleophilic oxygen atoms terminating the graphite (0001) surface abstract the formyl hydrogen and the activated aldehyde gets oxidized by epoxide-type mobile oxygen. Bielawski et al. [70] recently demonstrated the remarkable ability of graphene oxide to catalyze the oxidation of a variety of alcohols to corresponding ketones/aldehydes, alkenes to corresponding diones, and alkynes to corresponding hydrates — a process they termed as "carbocatalysis". These efficient metal-free reactions mark a promising convergence of heterogeneous catalysis with graphene science [71].

Liang et al. [72] reported that graphitic edges, presented in open cages of fullerene-like sites in glassy carbon, display high activity for the oxidative dehydrogenation of isobutane in comparison to the closed ones. Similar sites also play an important role to prepare very active Pt nanoparticles for PEM fuel cells [73]. On carbon nanotubes it is also possible to stabilize highly active dendritic [74] or flowerlike [75] Pt nanostructures. The area of the application of nanocarbon materials to prepare enhanced electrodes for PEM fuel cells, particularly for the oxygen reduction cathodic side, is one of the most active one in terms of publications. It is also emerging how the carbon plays a direct catalytic role in this process.

$\mathrm{Ni}$ et al. [76] showed that graphite-like nitrogen and Stone-Wales defect nitrogen decrease the energy barrier more efficiently than pyridine-like nitrogen, and a dissociation barrier lower than $0.2 \mathrm{eV}$ can be obtained. Higher graphite-like nitrogen concentration reduces the energy barrier much more efficiently due to partial occupation of $\pi^{*}$ orbitals and change of work functions. It was also shown how it is possible to prepare metal-free active oxygen reduction catalysts based on ordered mesoporous carbon nitrides with graphitic frameworks [77]. The role of graphitic edge plane exposure in carbon nanostructures for oxygen 
reduction reaction (ORR) was also discussed in detail by Biddinger and Ozkan [78]. Nanostructure on its own is not a factor for improved ORR activity. Rather, nanofibers with high edge plane exposure, like stacked platelets, provide the appropriate locations for $\mathrm{N}$ to incorporate into the graphitic matrix. Also gross $\mathrm{N}$ content did not play a role in ORR activity. $\mathrm{N}$ atoms have to be incorporated into the graphitic matrix, not attached as part of a surface functional group.

It is increasing the interest also on the use of nanocarbon materials for dye-sensitized solar cells [7981] and as advanced support for semiconductors such as $\mathrm{TiO}_{2}$ [82], $\mathrm{WO}_{3}$ [83] or $\mathrm{ZnO}$ [84] with enhanced visible-light photo-response. Carbon-modified $\mathrm{Bi}_{2} \mathrm{WO}_{6}$ nanostructures were also shown to have improved photocatalytic activity under visible light [85]. In general, there is a raising interest on noncovalent assembly of carbon nanotube-inorganic hybrids [86]. These hybrid architectures find potential applications in many fields ranging from energy storage and conversion, to catalysis, sensing, and medical diagnosis and treatment.

The use of nanocarbon materials to prepare bioelectrodes by supporting enzymes and/or microorganisms is another emerging area of development. Zhu et al. [87] developed microelectrodes based on well-aligned carbon nanostructures (nano-pore and nano-brush), and used them to support glucose oxidase enzyme. Ueda et al. [88] developed a carbon electrode with thornlike surface nanostructures to realize efficient direct electron transfer with enzymes for potential applications in biofuel cells. A conductive nanocage composed of palladium nanoparticles in mesocellular carbon-silica foam was developed by $\mathrm{Wu}$ et al. [89] to support glucose-oxidize enzyme and realize electrodes for biosensing and biomicroreactors. The role of carbon in these materials is important for biocompatibility and to control hydrophilicity.

Therefore, there is a range of new areas of development in the field of nanocarbon based catalytic materials, due to a better understanding of the surface nature of the functional sites and how to control the nano-architecture. The largest area of interest is still on the development of advanced nanostructured electrodes for applications ranging from energy storage (Li-ion batteries, supercapacitors) to energy conversion (fuel cells, solar devices). The use of nano-carbon materials as electrodes provides higher electrode-electrolyte contact area, faster charge transport, and novel possibilities for more efficient architecture. In PEM fuel cells they can realize efficient metal dispersion, improved stability and an optimal hierarchically-organized design [10,23]. However, it may be still considered their exploitation at the beginning stage. A better understanding of their key aspects and how they are linked to catalytic reactivity is required, to put on more rational bases for their use as advanced catalysts.

\section{Conclusions}

This introductory paper of the special issue of Catalysis Today dedicated to selected contributions presented at Carbocat-IV symposium overviews, from a personal and concise perspective, the trends and new opportunities offered from the use of nanocarbon based catalytic materials. This is a highly active field of development. This special issue is also the successor issue to "Carbon for Catalysis" in Catalysis Today, February 2010 [90], highlighting the most important developments of the last two years. New possibilities in terms of synthesis and understanding of the surface chemistry and nano-architecture have triggered the 
development of advanced applications, first in the area of nanostructured electrodes (which is still an active area of development) to extend progressively (although still slowly) to new sectors.

Although this contribution is not a systematic state-of-the-art description, but rather a critical selection and personal view, it provides suggestions for new possibilities, both scientific and applicative. It also demonstrates how this scientific area is becoming an increasingly relevant and important autonomous subject in heterogeneous catalysis, and how carbon offers an unmatched possibility for designing advanced catalysts. There is thus a bright future in catalysis by carbon based materials, even if active carbon may be considered one of the oldest supports for catalysis.

\section{References}

1. H. Juentgen, Fuel, 65 (1986) 1436.

2. F. Rodríguez-Reinoso, Carbon, 36 (1998) 159.

3. W.M.T.M. Reimerink, Studies in Surface Science and Catal., 120A (1999) 751.

4. V. Calvino-Casilda, A.J. Lopez-Peinado, C.J. Duran-Valle, R.M. Martin-Aranda, Catal. Reviews Science and Eng., 52 (2010) 325.

5. T.J. Bandosz, in Carbon Materials for Catalysis (eds P. Serp and J. L. Figueiredo), John Wiley \& Sons, Inc., Hoboken, NJ, USA. (2008) pp. 45-92, doi: 10.1002/9780470403709.ch2

6. D.S. Su, ChemSusChem, 4 (2011) 811.

7. S. Iijima, T. Ichihashi, Nature, 363 (1993) 603.

8. J.-P. Tessonnier, D.S. Su, ChemSusChem, 4 (2011) 824.

9. G. Centi, S. Perathoner, Catal. Today, 150 (2010) 151.

10. G. Centi, S. Perathoner, ChemSusChem, 4 (2011) 913.

11. T.N. Hoheisel, S. Schrettl, R. Szilluweit, H. Frauenrath, Angew. Chem. Int. Ed., 49 (2010) 6496.

12. D.E. Resasco, in Nanoscale Materials in Chemistry, 2nd Ed (eds K. J. Klabunde and R. M. Richards), John Wiley \& Sons, Inc., Hoboken, NJ, USA (2009). pp. 443-491. doi: 10.1002/9780470523674.ch13

13. J. Pyun, Angew. Chem. Int. Ed., 50 (2011) 46.

14. S.-S. Yu, W.-T. Zheng, Nanoscale, 2 (2010) 1069.

15. L.S. Panchakarla, A. Govindaraj, C.N.R. Rao, Inorg. Chimica Acta, 363 (2010) 4163.

16. S.R. Stoyanov, A.V. Titov, P. Kral, Coord. Chem. Rev., 253 (2009) 2852.

17. P. Ayala, R. Arenal, M. Ruemmeli, A. Rubio, T. Pichler, Carbon, 48 (2010) 575.

18. S.-H. Lee, D.-H. Lee, W.-J. Lee, S.-Ouk K., Adv. Funct. Mat., 21 (2011) 1338.

19. Y. Lei, K.-S. Yeong, J.T.L. Thong, W.-K. Chim, Chem. Mater., 16 (2004) 2757.

20. I. Janowska, S. Hajiesmaili, D. Begin, V. Keller, N. Keller, M.-J. Ledoux, C. Pham-Huu, Catal. Today, 145 (2009) 76.

21. W. Xiong, F. Du, Y. Liu, A. Perez, M. Supp, T.S. Ramakrishnan, L. Dai, L. Jiang, J. Am. Chem. Soc., 132 (2010) 15839.

22. Y. Liu, I. Janowska, T. Romero, D. Edouard, L.D. Nguyen, O. Ersen, V. Keller, N. Keller, C. Pham-Huu, 
Catal. Today, 150 (2010) 133.

23. G. Centi, S. Perathoner, Eur. J. Inorg. Chem., 26 (2009) 3851.

24. D.S. Su, G. Centi, in Nanoporous Materials for Energy and the Environment, (G. Rios, G. Centi, N. Kanellopoulos ed.s), Pan Stansford Pub. Singapore (2012), pp. 173-202.

25. D.S. Su, J. Zhang, B. Frank, A. Thomas, X. Wang, J. Paraknowitsch, R. Schlögl, ChemSusChem, 3 (2010) 169.

26. D. Yu, E. Nagelli, F. Du, L. Dai, J. Phys. Chem. Lett., 1 (2010) 2165.

27. D.S. Su, X.W. Chen, Angew. Chem. Int. Ed., 46 (2007) 1823.

28. M. Endo, K. Takeuchi, Y. Kim, K. Park, T. Ichiki, T. Hayashi, T. Fukuyo, S. Iinou, D. Su, M. Terrones, M. Dresselhaus, ChemSusChem, 1 (2008) 820.

29. X.-W. Chen, O. Timpe, S.B.A. Hamid, R. Schlögl, D.S. Su, Carbon, 47(2009) 340.

30. S.J. Guo, S.J. Dong, Chem.Soc. Rev., 40 (2011) 2644.

31. N.L. Rangel, J.C. Sotelo, J.M. Seminario, J. Chem. Phys., 131 (2009) 031105/1.

32. I. Janowska, O. Ersen, T. Jacob, P. Vennégues, D. Bégin, M.-Ja. Ledoux, C. Pham-Huu, Appl. Catal. A: General, 371 (2009) 22.

33. M.-S. Wang, Y. Bando, J.A. Rodriguez-Manzo, F. Banhart, D. Golberg, ACS Nano, 3 (2009) 2632.

34. C. Tao, L. Jiao, O.V. Yazyev, Y-C. Chen, J. Feng, X. Zhang, R.B. Capaz, J.M. Tour, A. Zettl, S.G. Louie, Nature Physics, 7 (2011) 616.

35. F.R. Negreiros, E. Apra, G. Barcaro, L. Sementa, S. Vajda, A.Fortunelli, Nanoscale, 4 (2012) 1208.

36. C.D. Liang, Z.J. Li, S. Dai, Angew. Chem. Int. Ed., 47 (2008) 3696.

37. Y. Meng, D. Gu, F. Zhang, Y. Shi, H. Yang, Z. Li, C. Yu, B. Tu, D. Zhao, Angew. Chem. Int. Ed., 44 (2005) 7053.

38. C.D. Liang, S. Dai, J. Am. Chem. Soc., 128 (2006) 5316.

39. C.H. Huang, R-a. Doong, D. Gu, D. Zhao, Carbon, 49 (2011) 3055.

40. C.H. Huang, Q. Zhang, T.-C. Chou, C.-M. Chen, D.S. Su, R.-A. Doong, ChemSusChem, (2012), in press. doi: $10.1002 / \operatorname{cssc} .201100618$.

41. J.J. Vilatela, D. Eder, ChemSusChem, (2012) in press. doi: 10.1002/cssc.201100536

42. H.-L. Ma, D.S. Su, G.-Q. Jin, X.-Y. Guo, Carbon, 44 (2006) 2254.

43. D.S. Su, R. Schlögl, ChemSusChem, 3 (2010) 136.

44. D.S. Su, X. Chen, G. Weinberg, A. Klein-Hofmann, O.Timpe, S. Bee Abd. Hamid, R. Schlögl, Angew. Chem. Int. Ed., 44 (2005) 5488.

45. J.-P. Tessonnier, D. Rosenthal, T. W. Hansen, C. Hess, M. E. Schuster, R. Blume, F. Girgsdies, N. Pfänder, O. Timpe, D. S. Su, R. Schlögl, Carbon, 47 (2009) 1779.

46. R. Arrigo, M.E. Schuster, S. Wrabetz, F. Girgsdies, J.-P. Tessonnier, G. Centi, S. Perathoner, D.S. Su, R. Schlögl, ChemSusChem, (2012), in press. doi: 10.1002/cssc.201100641.

47. G. Centi, S. Perathoner, ChemSusChem, 3 (2010) 195.

48. C. Ampelli, G. Centi, R. Passalacqua, S. Perathoner, Energy \& Env. Science, 3 (2010) 292. 
49. E. Castillejosa, B. Bachiller-Baezaa, I. Rodríguez-Ramosa, A. Guerrero-Ruiz, Carbon, (2012), in press. doi: 10.1016/j.carbon.2012.02.032.

50. B. Frank, S. Wrabetz, O.V. Khavryuchenko, R. Blume, A. Trunschke, R. Schlögl, ChemPhysChem, 12 (2011) 2709.

51. R., Arrigo, M. Hävecker, R. Schlögl, D.S. Su, Chem. Comm., 40 (2008) 4891.

52. R. Arrigo, M. Hävecker, S. Wrabetz, R. Blume, M. Lerch, J. McGregor, E.P. Parrott, J.A. Zeitler, L.F. Gladden, A. Knop-Gericke, R. Schlögl, D.S. Su, J. Am. Chem. Soc., 132 (2010) 9616.

53. M. Salmeron, R. Schlog1, Surface Science Reports, 63 (2008) 169.

54. J. Zhang, X. Liu, R. Blume, A. Zhang, R. Schlögl, D.S. Su, Science, 322 (2008) 73.

55. D.S. Su, T. Jacob, T.W. Hansen, D. Wang, R. Schlögl, B. Freitag, S Kujawa, Angew. Chem. Int. Ed., 47 (2008) 5005.

56. J. Kotakoski1, A.V. Krasheninnikov, U. Kaiser, J.C. Meyer, Phys. Rev. Lett., 106 (2011) 105505-1.

57. L.R. Radovic, J. Am. Chem. Soc., 131 (2009) 17166.

58. F. Banhart, Nanoscale, 1 (2009) 201.

59. L.-L. Wang, S. V. Khare, V. Chirita, D. D. Johnson, A. A. Rockett, A. I. Frenkel, N. H. Mack, R. G. Nuzzo, J. Am. Chem. Soc., 128 (2006) 131.

60. W. Qin, X. Li, Chem. Phys. Lett., 502 (2011) 96.

61. T. Zoberbier, T.W. Chamberlain, J. Biskupek, N. Kuganathan, S. Eyhusen, E. Bichoutskaia, U. Kaiser, A.N. Khlobystov, J. Am. Chem. Soc., 134 (2012) 3073.

62. Y-A. Lv, Y-H. Cui, X-N. Li, X-Z. Song, J-G. Wang, M. Dong, Physica E: Low-Dimensional Systems \& Nanostructures, 42 (2010) 1746.

63. K. Kwon, S-a. Jin, C. Pak, H. Chang, S.H. Joo, H.I. Lee, J.H. Kim, J.M. Kim, Catal. Today, 164 (2011) 186.

64. B. Shan, K. Cho, Chem. Phys. Lett., 492 (2010) 131.

65. H.O. Seo, K-D. Kim, M-G. Jeong, Y.D. Kim, K.H. Choi, E.M. Hong, K.H. Lee, D.C. Lim, Macromolecular Res., 20 (2012) 216.

66. H-Y. Lee, W. Vogel, P. Po-Jen Chu, Langmuir, 27 (2011) 14654.

67. M. Soria-Sanchez, A. Maroto-Valiente, J. Alvarez-Rodriguez, V. Munoz-Andres, I. Rodriguez-Ramos, A.Guerrero-Ruiz, Appl. Catal., B: Env., 104 (2011) 101.

68. E.J.Yoo, T. Okada, T. Akita, M. Kohyama, I. Honma, J. Nakamura, J. Power Sources, 196 (2011) 110.

69. B. Frank, R. Blume, A. Rinaldi, A. Trunschke, R.Schlögl, DGMK Tagungsbericht, 2011-2(Preprints of the DGMK-Conference "Catalysis: Innovative Applications in Petrochemistry and Refining", 2011), (2011) 211.

70. D.R. Dreyer, C.W. Bielawski, Chem. Sci., 2 (2011) 1233.

71. J. Pyun, Angew. Chem. Int. Ed., 50 (2011) 46.

72. C. Liang, H. Xie, V. Schwartz, J. Howe, S. Dai, S.H. Overbury, J. Am. Chem. Soc., 131 (2009) 7735.

73. B. Seger, P.V. Kamat, J. Phys. Chem. C, 113 (2009) 7990. 
74. M. Sanles-Sobrido, M.A. Correa-Duarte, S. Carregal-Romero, B. Rodriguez-Gonzalez, R.A. AlvarezPuebla, P. Herves, L.M. Liz-Marzan, Chem. of Mater., 21 (2009) 1531.

75. S. Ghosh, C.R. Raj, J. Phys. Chem. C, 114 (2010) 10843.

76. S. Ni, Z. Li, J. Yang, Nanoscale, 4 (2012) 1184.

77. K. Kwon, Y.Ji. Sa, J.Y. Cheon, S.H. Joo, Langmuir, 28 (2012) 991.

78. E.J. Biddinger, U.S.Ozkan, J. Phys. Chem. C, 114 (2010) 15306.

79. M. Wu, X. Lin, L. Wang, W. Guo, Y. Wang, J. Xiao, A. Hagfelde, T. Ma, J. Phys. Chem. C, 115 (2011) 22598.

80. A. Mathew, G.M. Rao, N. Munichandraiah, Mat. Res. Bull., 46 (2011) 2045.

81. B. Fang, S-Q. Fan, J.H. Kim, M-S. Kim, M. Kim, N.K. Chaudhari, J. Ko, J-S. Yu, Langmuir, 26 (2010) 11238 .

82. L. Tian, L.Ye, K. Deng, L. Zan, J. Solid State Chem., 184 (2011) 1465.

83. X. Zhang, X. Lu, Y. Shen, J. Han, L. Yuan, L. Gong, Z. Xu, X. Bai, M. Wei, Y. Tong, Chem. Comm., 47 (2011) 5804.

84. S. Cho, J-W. Jang, J.S. Lee, K-H. Lee, CrystEngComm, 12 (2010) 3929.

85. Y. Li, J. Liu, X. Huang, J. Yu, Dalton Trans., 39 (2010) 3420.

86. X. Li, Y. Qin, S.T. Picraux, Z-X. Guo, J. Mat. Chem., 21 (2011) 7527.

87. Z. Zhu, L. Garcia-Gancedo, A.J. Flewitt, F. Moussy, Y. Li, W.I. Milne, J. Chem. Techn. and Biotechn., 87 (2012) 256.

88. A. Ueda, D. Kato, R. Kurita, T. Kamata, H. Inokuchi, S. Umemura, S. Hirono, O. Niwa, J. Am. Chem. Soc., 133 (2011) 4840.

89. S. Wu, J. Wu, Y. Liu, H. Ju, Chem. of Mater., 20 (2008) 1397.

90. D.S. Su, S. Perathoner, H. Bitte, Catalysis Today, 150 (2010) 1.

Table 1 SciFinder number of item found (as concept, English only) for years 1990, 2000 and 2010 for different keywords.

\begin{tabular}{ccccc}
\hline \multirow{2}{*}{ Year } & \multicolumn{5}{c}{ Keywords: catalysis AND .... } \\
& carbon & nano carbon & carbon electro & carbon photo \\
\hline 1990 & 1244 & 0 & 112 & 30 \\
2000 & 2713 & 232 & 270 & 42 \\
2010 & 7222 & 2135 & 1268 & 324 \\
\hline
\end{tabular}

